\title{
Surgical Treatment of a Patient with See-Saw Nystagmus (SSN): Case Report
}

\author{
Lelio Sabetti*, Gianluca Murano, Francesca Guetti \\ Department of Biotechnological and Applied Clinical Sciences, University of L'Aquila, L'Aquila, Italy \\ Email: *studiosabetti@yahoo.it
}

How to cite this paper: Sabetti, L., Murano, G. and Guetti, F. (2020) Surgical Treatment of a Patient with See-Saw Nystagmus (SSN): Case Report. Open Journal of Ophthalmology, 10, 283-287. https://doi.org/10.4236/ojoph.2020.104031

Received: August 7, 2020

Accepted: November 7, 2020

Published: November 10, 2020

Copyright (c) 2020 by author(s) and Scientific Research Publishing Inc. This work is licensed under the Creative Commons Attribution International License (CC BY 4.0).

http://creativecommons.org/licenses/by/4.0/

\begin{abstract}
Background: Acquired See-saw Nystagmus (SSN) is a rare form of nystagmus characterized by elevation and intorsion of one eye with synchronous depression and intorsion of the contralateral eye in the first half cycle, followed by a reversal in the direction of the movements during the next half cycle. We herein report a case of a 47-year-old woman with a 3-year history of constant diplopia as a consequence of multiple neurosurgical interventions due to hemorrhage from a cavernous angioma located in the subthalamic region. She also had a history of major depressive disorder and ulcerative colitis. The patient underwent a surgical intervention with a $5 \mathrm{~mm}$ bilateral recession of the superior and inferior rectus muscles. Five years after surgery, the patient reported less recurrent and prominent episodes of transient horizontal deviation with horizontal diplopia, with a prevalence of well-being and comfort.
\end{abstract}

\section{Keywords}

Case Report, See-Saw Nystagmus, Surgery, Vertical Muscles Recession, Abnormal Head Position (AHP)

\section{Introduction}

The earliest known description of see-saw nystagmus was in 1913 by Maddox [1]. In clinical practice, abnormal eye movements are frequently related to brain disorders. Indeed, the etiopathogenesis of SSN is associated with a variety of acquired forms concerned with the Central Nervous System: parasellar masses [2], multiple sclerosis [3], mesodiencephalic lesions [4], radiation treatments or intrathecal methotrexate [5], and more frequently trauma [6], may result in ophthalmological disorders. Anatomically, we distinguish between mesodiencephalic lesions that affect the interstitial nucleus of Cajal and vestibular lesions that produce jerk-waveform see-saw nystagmus known as hemi-seesaw nystagmus; 
whereas the pendular form is associated with lesions affecting the optic chiasm. The frequency of oscillation is lower in the pendular $(2-4 \mathrm{~Hz})$ than in the hemi-seesaw nystagmus.

The primary goal of the treatment of SSN is to suppress the ocular oscillations and therefore to reduce the subjective visual symptoms: blurred vision, diplopia and oscillopsia. Medical treatments alone (Ethanol, Clonazepam or Memantine) have not yet yielded encouraging results and the positive effects remain fairly limited. Indeed, several common side-effects such as headache, dizziness, drowsiness, incoordination, lethargy, have been reported. Numerous treatment approaches for other forms of nystagmus exist, including: Botulinum Toxin type A (the exotoxin released by Clostridium Botulinum acts at the presynaptic receptors in the neuromuscular junctions thereby inhibiting the release of acetylcholine and inducing reversible chemical denervation that plays a significant role in the treatment of both extraocular disorders, such as hemifacial spasm or spasticity, and in ocular disorders such as nystagmus); or with surgical interventions in patients affected by nystagmus with abnormal head position (AHP) accompanied by severely acute, persistent and ipsilateral torticollis, as well as considerably impairing difficulties with social interactions. Known surgical treatments of different types of nystagmus include the Kestenbaum-Anderson method (a recession-resection procedure of the four rectus muscles), the Anderson technique (involving only recession of medial and lateral rectus muscles), or the application of both procedures in patients with a torsional component, as in the case of SSN: correction can be performed by weakening of the oblique muscle with the Kestenbaum principle, the Bietti principle (all four horizontal recti are recessed, with a $10 \mathrm{~mm}$ recession of the medial recti and a $12 \mathrm{~mm}$ recession of the lateral recti, thus avoiding consecutive exotropia and leading to a reduction in the frequency of the jerking movement by $50 \%$ ), or with the most recent Dell' Osso-Hertle procedure [7] (tenotomy of the horizontal muscles, thereby the simple detachment of the horizontal recti and reattachment at the original insertion would damp the proprioceptive feedback and therefore the intensity of the jerk-like movements).

The aim of this study is to evaluate the results obtained in a case of SSN treated using a new surgical technique of recession of the four vertical muscles.

\section{Case Presentation}

A 47-year-old Caucasian female presented in December 2014 for evaluation of SSN with referred diplopia subsequent to multiple neurosurgical interventions undergone in 2012 as a cause of a cavernous angioma with intracranial hemorrhage located at the subthalamic region. Her past medical history was significant for major depressive disorder and ulcerative colitis. Her medications included 300-mg Lithium Carbonate tablets and 1200-mg Mesalazine tablets on a daily basis. The patient denied any past eye surgery, any ocular treatments and any known drug allergies. 
At the time of the first visit a comprehensive examination was done that included the following tests: Distance Visual Acuity (DVA), Intraocular Pressure (IOP), Slit-Lamp Exam and Fundus Examination. Results were considered within normal limits for her age for both eyes (OU). Abnormal head posture with alternating head tilt towards the right/left shoulders due to see-saw nystagmus was also reported. Nystagmus was characterized in the first half of the cycle by the elevation and torsion of one eye with synchronous depression and extra torsion of the other eye; in the other half of the cycle, the movement was reversed. Red Filter Test was performed at both testing distances (distance and near) revealed diplopia. The Alternate Prism Cover Test at near fixation in acute stage measured an exotropia (XT) of 14, and disclosed a 20 prism diopters (PD) OD/OS and right exotropia, which in remission stage was 16 OS/OD 5PD, and at distance, it measured 16PD OU. The patient reported diplopia, nausea and unstable fusion.

The prism correction resulted to be ineffective. The patient reported that one year before she had undergone botulinum toxin treatment at a different center, but with negative outcomes. Therefore, we opted for surgery to perform weakening of elevation, of depression, of intorsion and of extorsion. We planned the resection of the four vertical muscles that in their primary and secondary function have the elevation and intortion (superior rectus) or depression and extortion (inferior rectus), respectively.

Informed consent was obtained from the patient regarding the proposed surgical technique and the following publication of the case.

In April 2015, the patient underwent surgery with a $5 \mathrm{~mm}$ recession of the superior and inferior rectus muscles in OU. The first follow-up visit was performed post-surgery. The other follow-ups were conducted at 7 and 30 days, and at 3 months. On the first follow-up visit, a significant objective and subjective improvement was observed with nystagmus being almost completely suppressed. On the 5-year follow-up, in February 2020, complete examinations were repeated including the following tests: Distance Visual Acuity (DVA), Intraocular Pressure (IOP), Slit-Lamp exam and Fundus Examination. Findings were considered within normal limits in OU. No evidence of AHP was observed. See-saw nystagmus, whose periodic rhythmic oscillations occurred every 2 - 3 minutes preoperatively, after surgery was very occasional, with minor or absent jerk movements in resting phase. Exams showed no evidence of diplopia as well as absence of any jerking movement. The Alternate Prism Cover Test in acute stage disclosed esotropia (ET) of 12PD (horizontal diplopia), whereas in remission stage it revealed exophoria $\left(\mathrm{XF}_{\mathrm{r}} 4\right)$.

The patient, however, reported less prolonged and less recurrent manifestations of transient horizontal deviation with horizontal diplopia, with a prevalence of well-being and comfort. Evidence of mild and occasional episodes of torsional (rotary) nystagmus was observed, while no reports of subjective impairments (no oscillopsia) were recorded. The study and data collection con- 
formed to all local laws and were compliant with the principles of the Declaration of Helsinki.

\section{Discussion}

See-saw Nystagmus is a rare ocular and extremely disabling disorder due to severe oscillopsia. Although Thurtell has advocated the medical treatment of SSN with clonazepam, gabapentin or memantine [8] [9] [10], the results obtained so far have not been shown to be efficacious, particularly due to the side effects (e.g., highly impaired concentration), therefore medical therapy alone cannot be considered adequate or efficacious. The surgical procedure performed with the recession of the four vertical muscles is borrowed from the same technique involving that involves the maximum recession of all four horizontal rectus muscles applied on the nystagmus without null zone [11] [12]. The positive outcome obtained may be due to the exploitation of the primary and secondary action of the vertical muscles and to the deafferentation caused by the tenotomy and reattachment of the rectus muscles [13] [14] [15].

However, in our case, it is not possible to establish whether this results from the deafferentation or from the direct weakening of the muscle actions. The improvement reported by the patient in terms of suppression of ongoing nystagmus, the less recurrent oscillopsy (only every 10 - 15 days with a duration of few minutes), the increase in visual acuity, the improvement of posture by reduction of AHP, are also confirmed at the five-year follow-up visit. This has allowed the recovery of the patient's social and work activities. The 5-mm recession of the vertical rectus muscles has not caused any pattern deviation, any cylodeviation and secondary overactions, or any alteration in the eyelid movements.

\section{Conclusion}

The new technique of recession of the four vertical muscles has yielded positive outcomes and it may be employed as a recommended procedure in similar cases. It would be desirable to compare the surgical outcomes obtained by other researchers.

\section{Acknowledgements}

The author would like to thank Marta Fiorenza for the linguistic assistance.

\section{Conflicts of Interest}

The authors declare no conflicts of interest regarding the publication of this paper.

\section{References}

[1] Maddox, E.E. (1913) See-Saw Nystagmus with Bitemporal Hemianopia. Proceedings of the Royal Society of Medicine, 7, 12-13.

[2] Barton, J.J. (1995) Blink- and Saccade-Induced Seesaw Nystagmus. Neurology, 45, 
831. https://doi.org/10.1212/WNL.45.4.831

[3] Samkoff, L.M. and Smith, C.R. (1994) Seesaw Nystagmus in a Patient with Clinically Definite MS. European Neurology, 34, 228-229. https://doi.org/10.1159/000117044

[4] Halmagyi, G.M., Aw, S.T., Dehaene, I., Curthoys, I.S. and Todd, M.J. (1994) Jerk-Waveform See-Saw Nystagmus Due to Unilateral Meso-Diencephalic Lesion. Brain, 117, 789-803. https://doi.org/10.1093/brain/117.4.789

[5] Epstein, J.A., Moster, M.L. and Spiritos, M. (2001) Seesaw Nystagmus Following Whole Brain Intrathecal Methotrexate. Journal of Neuro-Ophthalmology, 21, 264-265. https://doi.org/10.1097/00041327-200112000-00007

[6] Eggenberger, E. (2002) Delayed-Onset Seesaw Nystagmus Posttraumatic Brain Injury with Bitemporal Hemianopsia. Annals of the New York Academy of Sciences, 956, 588-591.

[7] Dell'Osso, L.F., Hertle, R.W., Williams, R.W. and Jacobs, J.B. (1999) A New Surgery for Congenital Nystagmus: Effects of Tenotomy on an Achiasmatic Canine and the Role of Extraocular Proprioception. Journal of American Association for Pediatric Ophthalmology and Strabismus, 3, 166-182.

https://doi.org/10.1016/S1091-8531(99)70063-7

[8] Thurtell, M.J. and Brinkley, J.J. (2013) Treatment of Nystagmus and Saccadic Oscillations. http://eyerounds.org/tutorials/Nystagmus/

[9] Thurtell, M.J., Joshi, A.C., Leone, A.C., Tomsak, R.L., Kosmorsky, G.S., Stahl, J.S. and Leigh, R.J. (2010) Crossover Trial of Gabapentin and Memantine as Treatment for Acquired Nystagmus. Annals of Neurology, 67, 676-680. https://doi.org/10.1002/ana.21991

[10] Frisén, L. and Wikkelso, C. (1986) Posttraumatic Seesaw Nystagmus Abolished by Ethanol Ingestion. Neurology, 36, 841-844. https://doi.org/10.1212/WNL.36.6.841

[11] Cochin, J.P., Hannequin, D., Domarcolino, C., Didier, T. and Augustin, P. (1995) Intermittent See-Saw Nystagmus Abolished by Clonazepam. Revue Neurologique (Paris), 151, 60-62.

[12] Noorden, G.K. and Sprunger, D.T. (1991) Large Rectus Muscle Recessions for the Treatment of Congenital Nystagmus. Archives of ophthalmology, 109, 221-224.

https://doi.org/10.1001/archopht.1991.01080020067045

[13] Helveston, E.M., Ellis, F.D. and Plager, D.A. (1991) Large Recession of the Horizontal Recti for Treatment of Nystagmus. Ophthalmology, 98, 1302-1305. https://doi.org/10.1016/S0161-6420(91)32139-0

[14] Dell'Osso, L.F. (2002) Development of New Treatments for Congenital Nystagmus. Annals of the New York Academy of Sciences, 956, 361-379. https://doi.org/10.1111/j.1749-6632.2002.tb02834.x

[15] Dell'Osso, L.F., Orge, F.H., Jacobs, J.B. and Wang, Z.I. (2014) Fusion Maldevelopment (Latent/Manifest Latent) Nystagmus Syndrome: Effects of Four-Muscle Tenotomy and Re-Attachment. Journal of Pediatric Ophthalmology \& Strabismus, 51, 180-188. https://doi.org/10.3928/01913913-20140326-01

\section{Abbreviations}

See-saw Nystagmus (SSN), Prism Diopters (PD), Left eye (OS), Right eye (OD), Both eyes (OU). 